\title{
Analysis of Finite-Duration Wide-Band Frequency Sweep Signals for Ocean Tomography
}

\author{
Timothy F. Duda
}

\begin{abstract}
A group of amplitude and frequency modulated signals which generate narrow synthesized pulses are described. The pulse-compression properties of these signals should approach those of maximal $(M)$ sequence phase-modulated signals now commonly used in ocean experiments. These amplitude-tapered linear frequency-sweep (chirp) type signals should be accurately reproducible with most acoustic sources, since they have controllable limited- bandwidth frequency content and differentiable phase. The Doppler response of the signals is calculated using a wide-band approach, where the frequency shift from relative motion is not constant throughout the waveform. The resultant Doppler effect on the matched-filter output is a function of the signal duration. The signals are suitable for use with tunable resonant transducers, and have adequate Doppler response for use with Lagrangian ocean drifters.
\end{abstract}

\section{INTRODUCTION}

N THE 1980's and early 1990's ocean acoustic tomography experiments, which use pulse travel-time measurements to create sound-speed and current maps and to evaluate ocean processes [1]-[5], have used coded phase-modulation pulse compression techniques [6]. In that scheme, a carrier is phase modulated with special binary pseudorandom codes (M-sequences). Cross-correlation (matched filtering) of received signals with the transmitted waveform provides highresolution arrival-time estimates. For tomography, limited peak projector power requires coherent two-minute transmissions for reception at ranges of up to $3000 \mathrm{~km}$, and bandwidths of $100 \mathrm{~Hz}$ are desirable for travel-time resolution of order $10 \mathrm{~ms}$. The binary nature of the phase modulation demands rapid phase transitions, which in turn demand low-Q acoustic sources. Although the binary signals are optimized in numerous ways [7] and have theoretically unlimited temporal resolution, only approximations to the signals can be practically implemented because transducers do not have arbitrarily low $Q$ and can not make instantaneous phase transitions. Therefore, the transducer bandwidth, rather than the nature of the signal, ultimately determines the temporal resolution limit.

The fact that the ideal properties of $\mathbf{M}$-sequences may not be realized in practice provides partial motivation for this examination of other signaling schemes which approach the performance of $\mathbf{M}$-sequences, and may be advantageous under certain circumstances where $\mathrm{M}$-sequence transmission is not possible. An example is the case of a tunable resonant device,

Manuscript received June 29, 1992; revised January 6, 1993. This work was supported by the Woods Hole Oceanographic Institution and by the ONR under Contracts N-00014-91-J-1246 and N00014-92-J-1162. This is Woods Hole Oceanographic Institution Contribution 8070.

The author is with the Applied Ocean Physics and Engineering Department, Woods Hole Oceanographic Institution, Woods Hole, MA 02543

IEEE Log Number 9207648. which may be both broadband and efficient in the $50-300$ $\mathrm{Hz}$ range. High efficiency is required to reduce electrical requirements, such as large batteries or cables. Extending a narrow resonance peak across a desired band through tuning will require a signal which alters its frequency content over time. If the band is occupied sequentially, then the signal is the well-known frequency sweep or FM slide. Narrow-band frequency sweep signals (modeled accurately with a constant Doppler shift over the entire signal band) have been analyzed in detail ([8] provides a review). Amplitude modulated sweep signals, including wide-band, have been analyzed in less detail [9], [10].

In the following, we investigate the properties of signals suitable for use with a tunable resonant transducer. We concentrate on linear frequency sweep signals, amplitude modulated with smooth functions, which we call tapered linear frequency modulated (TLFM) signals. These signals have good pulse compression response when Doppler shifted (when used in moving reference frames) [9], [10], and the effect of Doppler is examined both theoretically and numerically. Characteristics of echolocation wide-band ambiguity functions have been examined for this class of signals [9], [11], in particular for Doppler tolerance. We add to those analyses by tailoring our discussion to the tomographic requirements of sidelobe reduction (for multipath discrimination) and joint travel-time/Doppler estimation. Sidelobe reduction is essentially a treatment of end effects, which are endemic to purely FM signals. The Doppler problem is closely related to the duration of the FM signal, so that we focus on the mitigation of finite-duration effects. Vespertillionid bats (Myotis mystacinus) [11] and little brown bats (Myotis lucifugus) [9] use Dopplertolerant tapered FM sweeps while cruising, presumably for location of insects, and are examples of evolutionary development of the TFM scheme (a generalization of TLFM).

The spectral content of synthetic pulse signals is discussed in Section II, where some knowledge of pulse compression is assumed. The TLFM sweep signals we propose for use are defined in Section III. These signals are shown in Section IV to be compatible with the probable Doppler shift effects of drifting instruments. Section V covers joint Doppler velocity/arrival-time estimation with sweeps. The last section is a summary of possible advances which can be made through use of tunable sources and moving receivers, both made possible by these sweep signals.

\section{SPECTRa of SyNTHETIC PUlse Signals}

The power spectrum of a transmitted signal is fundamentally related to achievable time resolution. The optimal temporal 


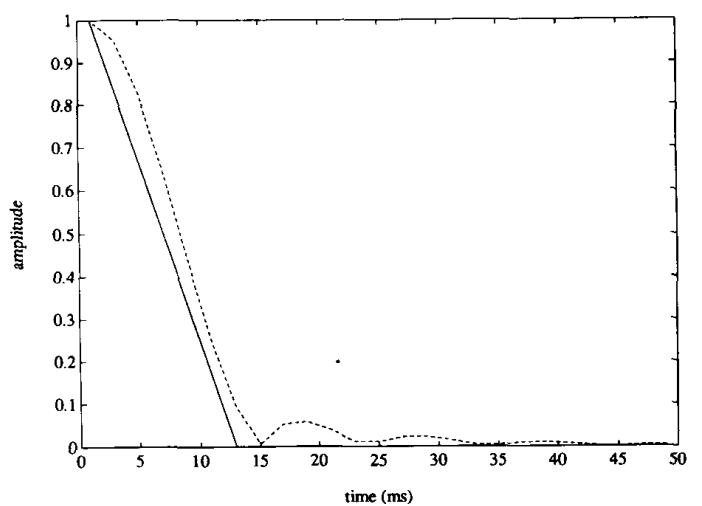

Fig. 1. Shown are one-half side of the matched-filter outputs for the ideal (solid) and filtered (dash) M-sequence phase-modulated signals. The truncation of frequencies gives the side-peaks.

pulse, the delta function, corresponds to a signal with a uniform power spectrum. Approximations to a delta function are achieved at long ranges in the ocean by synthesizing a short duration pulse through pulse compression of long, coded signals. Processing can be simple: individual multipath received signals are approximated by

$$
r(t)=s\left(t-t_{t}\right)+n(t)
$$

where $s(t)$ is a known transmitted signal and $t_{t}$ is the traveltime from source to receiver, and all other acoustic energy is regarded as noise $n$. The processed (matched filter) output

$$
R(\tau)=\left|\int_{-\infty}^{\infty} r(t) s^{*}(t-\tau) d t\right|
$$

is singly peaked at $\pi=t_{t}$ if $s(t)$ has a time-lagged autocovariance function $C(\tau)=\langle s(t) s(t-\tau)\rangle$ with a single peak at $\tau=0$.

In the pulse synthesis scheme, the time-domain correlation process is equivalent to frequency domain multiplication of the received signal spectrum and the complex conjugate of the transmitted signal, so that the matched-filter outputs are equal to inverse Fourier transforms of squares of the transmitted signal spectrum. Consider an example of $\mathrm{M}$-sequence phase modulation of a carrier. These signals, which have specific digit lengths corresponding to a small number of carrier cycles, have sinc $^{2}$ power spectra, the same spectra as rectangular pulses (digital pulses, in a sense). The sinc ${ }^{4}$ spectral product of the filtering procedure yields a triangular matched-filter output for the $\mathbf{M}$-sequence. A residual mean level results from the carrier energy and is a function of the modulation angle.

Fig. 1 shows the matched-filter output of a phase modulated $\mathrm{M}$-sequence code with a carrier frequency of $250 \mathrm{~Hz}$ and digit length of 3 cycles. If the spectrum of the $\mathrm{M}$-sequence signal is altered from a sinc ${ }^{2}$ spectrum by attenuating above 200 and below $300 \mathrm{~Hz}$ with a digital filter, the second (dashed) output results. As a result of the band limiting, the synthetic output pulse is widened and exhibits sidelobes.
The essential idea behind the TLFM pulse synthesis scheme is precise control of the signal spectrum, with resultant precise control of the synthesized pulse. Since the transmitted spectrum depends on the frequency response of physically realizable, bandwidth-limited devices, the signal must be modified accordingly, but this straightforward equalization step is not included in our analysis. A price one pays compared with phase modulation (including $\mathrm{M}$-sequence phase modulation) is lower average power in the signals, so that more time is required to achieve the output energy. This is an important consideration in a temporally fluctuating ocean environment.

\section{SPECTRAL CONTROl USING AMPLITUDE MODULATED SWEEPS}

We concentrate on linear frequency-sweep signals, with phase proportional to the square of time (plus an additional term concerning the center frequency):

$$
s(t)=A(t) e^{i \theta(t)} ; \quad \theta(t)=\frac{b t^{2}}{2}+\omega_{o} t
$$

Time-varying amplitude modulation can be used to precisely control frequency content, since the frequency content of the signal is also a function of time. For our purposes it is satisfactory to approximate the time-varying frequency content by a simple estimate of the instantaneous frequency, $f(t)=$ $d \theta / d t=b t+\omega_{\mathrm{o}}$. In this approximation, the power spectral density $\mathrm{S}$ of the signal $s$ follows the inverse formula

$$
S(f)=A^{2}(g(f))
$$

where frequency is mapped to time by $g(f)=\left(f-\omega_{o}\right) / b$, the inverse function of $f(t)$. Since $g$ is a linear function of frequency, the spectral density $S(f)$ has the same shape as $A^{2}(t)$.

The above relation has been checked numerically. Spectral errors $E(f)=S(f) / S\left(f_{o}\right)-A^{2}(f) / A^{2}\left(f_{o}\right)$ have been calculated for 15 -second long sweeps through bandwidths of 25 , 50,100 and $200 \mathrm{~Hz}$. The center frequency $f_{o}$ is $250 \mathrm{~Hz}$. Both Hanning and cosine tapers were employed for $A(t)$. Fig. 2a shows that fractional spectral errors for the Hanning taper have maxima and minima which are independent of sweep rate. At the slower sweep rate, $E$ shows a slight ringing at the band edges, which diminishes as the sweep rate (the bandwidth) increases. Fig. $2 \mathrm{~b}$ shows $E$ for cosine tapering. The band-edge ringing is increased by two orders of magnitude over that from the Hanning taper, dominating the spectral error. The ringing and the total error are worst for the slowest sweep, exceeding $0.13 \%(0.0056 \mathrm{~dB})$. For comparison, Fig. 3 shows $E$ for an untapered $(A=1), 15$ second, $100 \mathrm{~Hz}$ sweep. The superior performance of the Hanning taper can be attributed to smooth transitions at the ends of the sweep (positive second derivatives of $A$ ). Spectral errors, mostly at the band edges, will produce slight deviations from theoretical matched-filter output. These deviations are usually of no consequence, and can be reduced by careful choice of taper and sweep rate, if required.

Spectral estimation tapers, such as Hanning windows, are ideal $A(t)$ for TLFM since they are designed to reduce sidelobes in the Fourier transform domain [12]. For nonlinear 


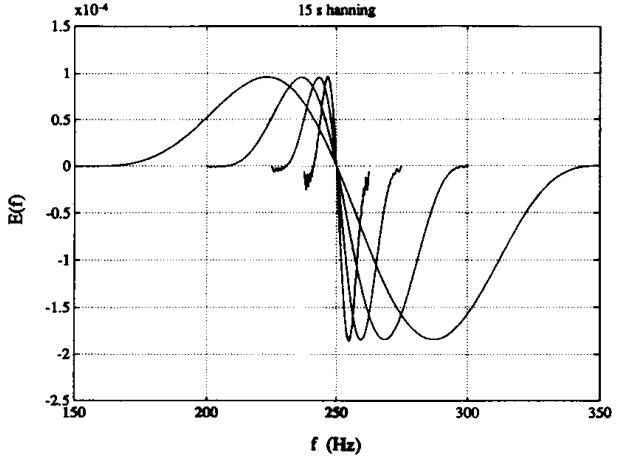

(a)

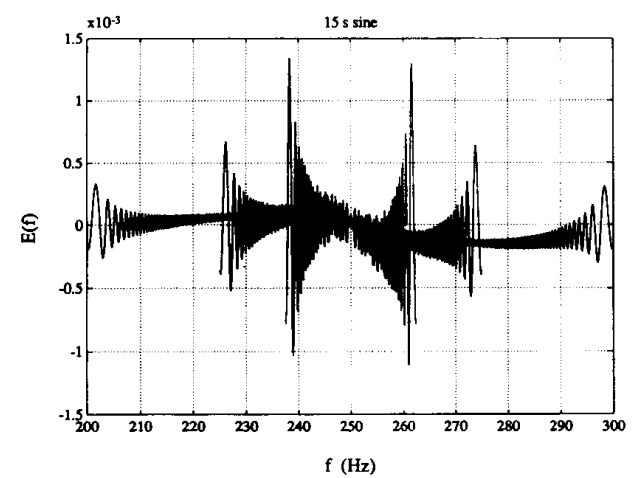

(b)

Fig. 2. Errors for normalized spectra, $E(f)$, for various sweep rates (that is, various bandwidths), for Hanning and cosine taper shapes. The center frequency is $250 \mathrm{~Hz}$, and the sweep duration is $15 \mathrm{~s}$. Frame (a) shows errors for four sweep rates using a Hanning taper. Frame (b) shows errors for three sweep rates using a cosine taper, which are larger than those for the Hanning taper.

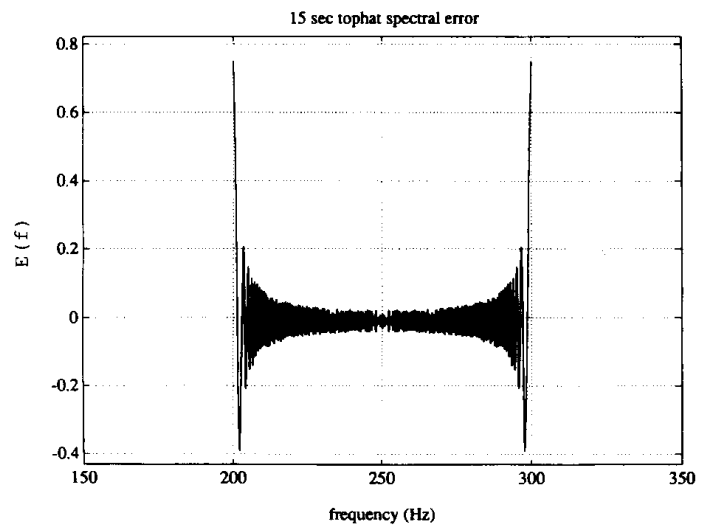

Fig. 3. The normalized spectral error $E(f)$ for the boxcar taper is orders of magnitude larger than for the cosine tapers (Fig. 2).

FM, appropriate $A$ can be calculated using the tapers and the inverse spectral formula. Transmission of tapered sweeps and pulse synthesis using (2) has been referred to as bilateral

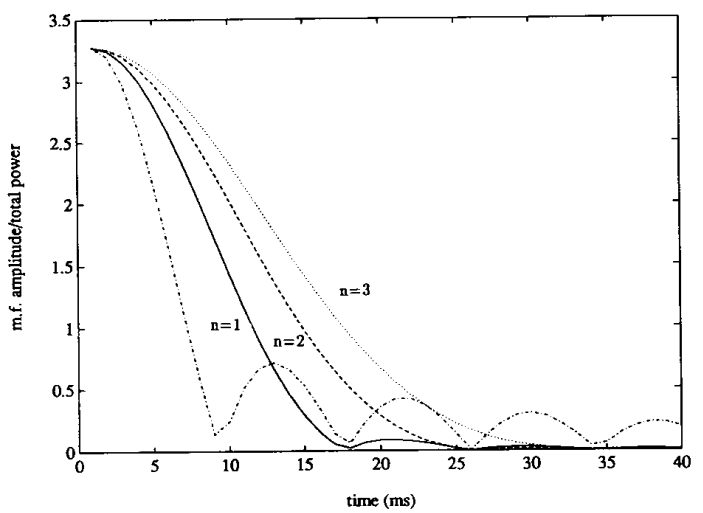

Fig. 4. Matched-filter outputs for TLFM using various taper functions $A(t)$. The total swept band is $120 \mathrm{~Hz}$. The narrowest main peak is produced by the boxcar function (no amplitude modulation). The solid line is the result of cosine tapering, the dashed from $\cos ^{2}$, and the dotted (widest peak) is from $\cos ^{3}$ tapering.

weighting [8, ch. 7]. A set of examples is cosine-taper modulation, $A(t)=\cos ^{n}(t) ; n=2$ corresponds to the Hanning taper. In the absence of noise or Doppler shifts, sidelobes and pulse width are not influenced by the frequency rate-ofchange $b$ (or the total transmisison time), only by the total range of frequencies. Fig. 4 shows synthetic pulse shapes (matched filter) for $n=1,2,3$ and for boxcar modulation (i.e. $A(t)=1)$, with a bandwidth of $120 \mathrm{~Hz}$. Since different tapers result in different transmitted power, matched-filter outputs are normalized by transmitted power. The matched filtering involves the squaring of the frequency response $S(f)$, so that $n=1$ modulation gives a pulse shape equal to the transform of the Hanning taper $(n=2)$, and so on, as explained in Section II.

The boxcar produces the narrowest peak and the highest sidelobes. Greater distribution of energy over the available band produces the narrowest peak, but the sharp band-edge cutoffs produce ringing (sidelobes). The cosine ${ }^{\mathrm{n}}$ tapers reduce the sidelobes, but broaden the peak considerably. These tapers are often used since they are simple analytic forms, and do reduce sidelobes, but they are not optimal. There are many taper families offering comparable sidelobe reduction with less widening of the main lobe [12]. The functions that have the highest ratio of main-lobe energy for a given peak width are the prolate spheroidal functions [12], [13]. For ocean tomography, the issue of multipath discrimination may overshadow the requirement of gain, and rejection of nearby sidelobe energy may be more important than rejection of total sidelobe energy. Very good taper functions in this situation are the Chebyshev tapers. these are conveniently available in the MATLAB signal-processing software package, which calculates Chebyshev tapers with any desired sidelobe level [14]. To create synthetic pulses with the known sidelobe characteristics of the Chebyshev tapers, one can use amplitude functions $A(t)$ following the square-root of Chebyshev tapers. Fig. 5 shows synthetic pulse (matched-filter) output for seven rootChebyshev tapers with maximum sidelobes of -14 to $-32 \mathrm{~dB}$. 


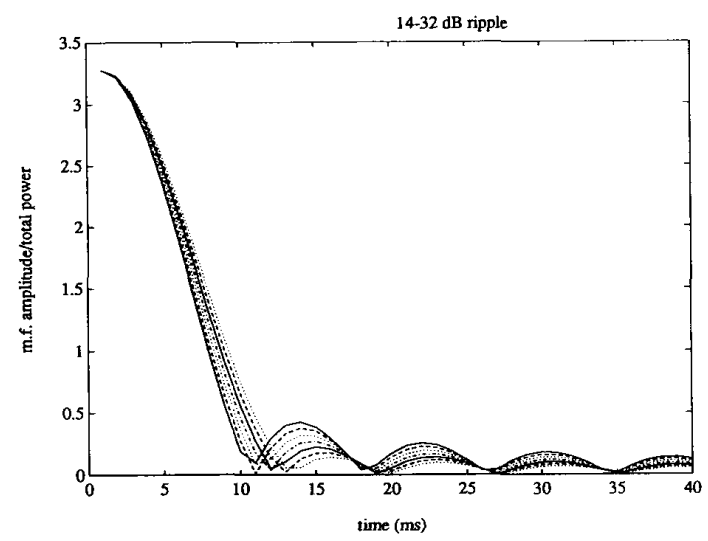

Fig. 5. Matched filter outputs using Chebyshev taper signals over a $120 \mathrm{~Hz}$ swept band. A continuous choice of main peak width vs. side peak energy is available. The performance is better than the cosine tapers, with a distribution of energy into many small sidelobes allowing a narrower main peak.

These maximum sidelobes are roughly equivalent to observed relative noise levels for $1000 \mathrm{~km}$ ocean transmission with typical radiators and 2-minute codes, and are thus adequate for complete multipath identification. The main lobes are not quite as narrow as the with ideal M-sequence (Fig. 1) or boxcar LFM (Fig. 4), but are similar to the band-limited M-sequence (Fig. 1).

\section{DOPPLER EFFECTS ON PULSE SYNTHESIS}

A nonzero relative velocity between source and receiver will result in a received signal with a compressed or stretched time scale relative to the zero-velocity case. For small velocity, a good approximation to the total length of the reception is

$$
T_{d}=T /(1+v / c) \approx T(1-v / c)
$$

where $c$ the wave speed, $v \ll c$ is the relative closing velocity, and $T$ is the duration of the transmission. Any transmitted frequency $\omega$ will arrive at the approximate shifted frequency

$$
\omega_{d}=\omega(1+v / c)
$$

The effect on signal coherence can be estimated if one considers the difference between $s(t-v t / c)$ and $s(t)$. At the total duration $T$, the signal mismatch is maximum. Conventionally, it is argued that a signal with bandwidth $W$ cannot change much in time $W^{-} 1$, so that Doppler effects are minimal if [15]

$$
\frac{v T}{c} \ll W^{-1}
$$

or

$$
W T \ll \frac{c}{v}
$$

This time-bandwidth product criterion is very stringent for typical ocean tomographic transmissions, which must be long to achieve gain. M-sequence codes of $120 \mathrm{~s}$ duration and $83 \mathrm{~Hz}$ bandwidth, such as used in the 1989 Slice89 experiment [16],

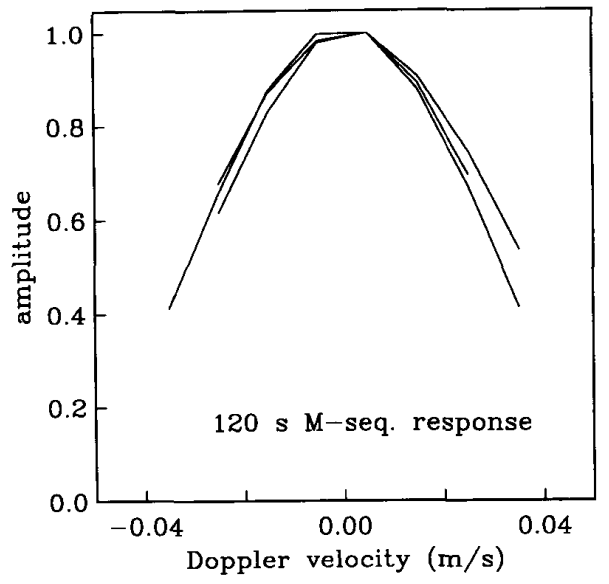

Fig. 6. Amplitude response of the matched filter for the 2-minute M-sequence signals used in a 1989 experiment (Slice89). The signals were phase modulated 1023 -digit sequences of $12.176 \mathrm{~s}$ duration, 3 cycles (of $250 \mathrm{~Hz}$ ) per digit, repeated 10 times. The data was investigated with an interpolative resampling matched-filter program provided by $\mathbf{K}$. Metzger of the University of Michigan. The resampling process imitates the effect of a moving receiver.

lose coherence at about $v=0.05 \mathrm{~m} / \mathrm{s}$, or 0.1 knots Doppler. The lost coherence results in digit mismatch and consequent attenuation of the matched filter output. Fig. 6 shows the effect on the matched-filter output when the sampling frequency is modified to simulate a Doppler-shifted $\mathbf{M}$-sequence reception. There is a peak-amplitude attenuation of over $50 \%$ at $v=0.04$ $\mathrm{m} / \mathrm{s}$. It is important to point out that Doppler sensitivity (or velocity discrimination) of signals such as $\mathrm{M}$-sequences is useful and important in some applications. Doppler-sensitive signals are not attenuated if a processing receiver can adjust the sampling frequency to provide a peak, thus jointly estimating $v$ and arrival time.

The smooth phase (or frequency) variation of the TLFM signals result in less decoherence between a compressed reception and a non-compressed replica than in the case of M-sequences. This is because the frequency sweep rate is affected only slightly and the amplitude modulation is very gradual. The result is performance in excess of the WT product criterion. Although optimally Doppler-tolerant tapered linearperiod FM signals exist [9], [11], we show here that TLFM signals with simple and optimal sidelobe control have adequate Doppler tolerance. (Extension of the analysis to the optimallyDoppler tolerant signals is straightforward.)

As discussed in Section II, the function $R(\tau)$ will peak at $\tau=t_{t}$ if $v=0$, since in that case $s(t-\tau)$ is very similar to $r(t)$. On the other hand, if $v$ is nonzero, the peak of $R(r, v)$ will not be at $\tau=t_{t}$ and the shape of $R(\tau, v)$ will change from that of $R(\tau, 0)$.

We will analyze two expressions for $R(\tau, v)$, and show how their behavior is related. The first is for narrow-band signals, and is nearly identical to Woodward's ambiguity function analysis for radar or sonar echolocation [8], [15]. The second is similar to the wide-band ambiguity function [8]-[11]. In each case we estimate the value of $\tau$ where $R(\tau, v)$ is maximum for specified $v$. 
For narrow-band FM sweeps, where the Doppler shift $\omega_{d}-\omega=\omega_{a}=f(v)$ can be considered constant over the band, the function $R$ can be approximated as

$$
\begin{aligned}
R_{n}(\tau, v)= & \mid \int_{-\infty}^{\infty} A\left(t-t_{t}\right) A(t-\tau) \\
& \cdot e^{i\left[\omega_{0}\left(t-t_{t}\right)+\omega_{a}\left(t-t_{t}\right)+b\left(t-t_{t}\right)^{2} / 2\right]} \\
& \cdot e^{-i\left[\omega_{o}(t-\tau)+b(t-\tau)^{2} / 2\right]} d t \mid
\end{aligned}
$$

Shifting to the perturbation domain $\tau^{\prime}=\tau-t_{t}, p=t-t_{t}$, and rearranging,

$$
R_{n}\left(\tau^{\prime}, v\right)=\left|e^{i\left[\omega_{\mathrm{o}} \tau^{\prime}-\tau^{\prime 2} b / 2\right]} \int_{-\infty}^{\infty} A(p) A\left(p-\tau^{\prime}\right) e^{i p\left[\omega_{a}+b \tau^{\prime}\right]} d p\right|
$$

The stationary-phase condition of the integrand is satisfied if $\omega_{a}+b \tau^{\prime}=0$, or $\tau_{n}^{\prime}=-\omega_{a} / b$. If $\omega_{a}$ is taken to be the perturbation at the mid-frequency $\omega_{c}=\omega_{o}+b T / 2$, the result for the $\tau^{\prime}$ of maximum $R_{n}$ is

$$
\tau_{n}^{\prime}=-\frac{\omega_{c} \epsilon}{b}
$$

where $\epsilon=v / c$.

For increased bandwidth the frequency shift can not be considered constant, and we instead consider the time dilation. $R$ takes a form similar to the wide-band ambiguity function,

$$
R_{b}(\tau, v)=\left|\int_{-\infty}^{\infty} r(t(1+\epsilon)) s^{*}(t-\tau) d t\right|
$$

where an amplification factor which results from time dilation and energy conservation has been neglected. Equation (12) can be rewritten

$$
\begin{aligned}
R_{b}(\tau, v)= & \mid \int_{-\infty}^{\infty} A\left(\left(t-t_{t}\right)(1+\epsilon)\right) A(t-\tau) \\
& \cdot e^{i \theta\left(\left(t-t_{t}\right)(1+\epsilon)\right)} e^{-i \theta(t-\tau)} d t \mid
\end{aligned}
$$

Relocating the integral $p=t-t_{t}-T / 2$ and $\tau^{\prime}=\tau-t_{t}$, and retaining terms to first order in $\epsilon$,

$$
\begin{aligned}
R_{b}\left(\tau^{\prime}, v\right)= & \mid e^{i\left[\omega_{o}\left(\tau^{\prime}+\epsilon T / 2\right)+\left(\epsilon T^{2} / 2+T \tau^{\prime}-\tau^{\prime 2}\right) b / 2\right]} \\
& \cdot \int_{-\infty}^{\infty} A((p+T / 2)(1+\epsilon)) A\left(p+T / 2-\tau^{\prime}\right) e^{i \epsilon b p^{2}} \\
& \cdot e^{i p\left[\epsilon \omega_{\circ}+\epsilon b T+b \tau^{\prime}\right]} d p \mid
\end{aligned}
$$

Comparison with (10) shows a slightly different stationary phase point. The function $\exp \left(i \epsilon b p^{2}\right)$ is slowly varying

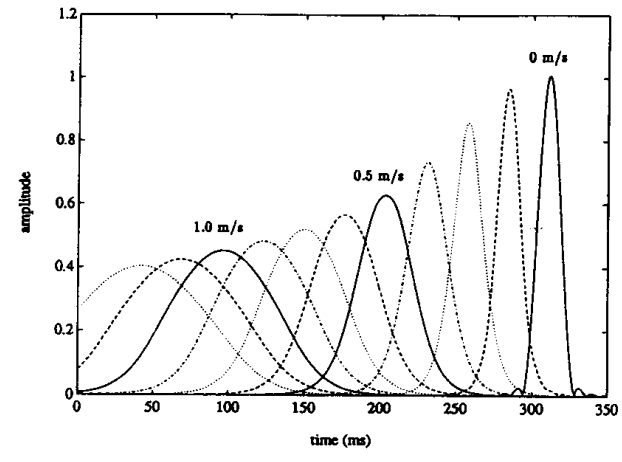

(a)

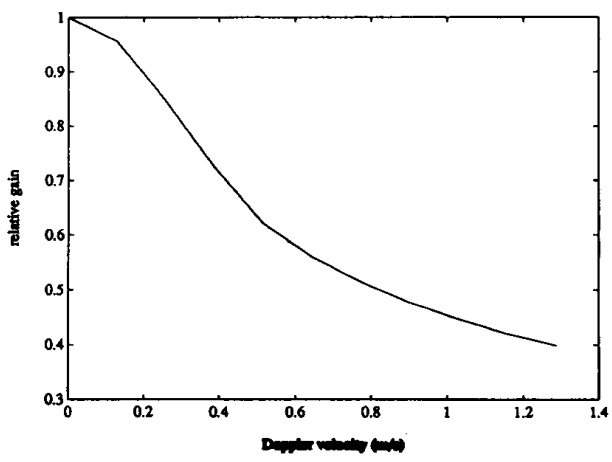

(b)

Fig. 7. (a) Synthetic pulse (matched filter) results of a relative source-receive motion simulation using a two minute, $120 \mathrm{~Hz}$ band cosine-taper TLFM signal. The step in velocity $v$ is $0.125 \mathrm{~m} / \mathrm{s}$, from 0 to $1.25 \mathrm{~m} / \mathrm{s}$. A two minute M-sequence signal would produce only a large $v=0$ peak and possibly a small $v=0.125 \mathrm{~m} / \mathrm{s}$ peak (fig. 6), while this TLFM signal gives wide but visible peaks to over $1 \mathrm{~m} / \mathrm{s}$. Frame (b) shows the peaks heights from frame (a), relative to the $v=0$ case, to be compared with Fig. 6 .

throughout the range of nonzero, $A$, approximately $-T / 2<$ $p<T / 2$ if $\epsilon b$ is sufficiently small, and its effects is neglected. The stationary phase condition can be written

$$
\tau_{b}^{\prime}=-\left(\omega_{o}+\frac{b T}{2}\right) \frac{\epsilon}{b}-\frac{\epsilon T}{2}
$$

where the first term is recognized as the narrow-band solution of (11).

The location of the wide-band $R$ peak is approximately the sum of the narrow-band $\tau_{n}^{\prime}$ and a correction related only to the finite signal duration. The correction is $\delta T / 2=-T \epsilon / 2$, where $\delta T$ is the change in length of the received waveform due to Doppler. It is useful to think of $\delta T / 2$ as the average time shift of the received waveform due to its Doppler length alteration from $T$ to $T+\delta T$ by Doppler.

Fig. 7 shows the Doppler performance for a 120 s, 120 $\mathrm{Hz}$, amplitude-modulated up-sweep in frequency. As Doppler velocity is increased, the peak spreads and looses amplitude, but does not split. The "toward," or "blue" Doppler shifts give the progressively earlier apparent arrival times. Fig. 7 also shows peak attenuation vs. closing velocity. In the previous 


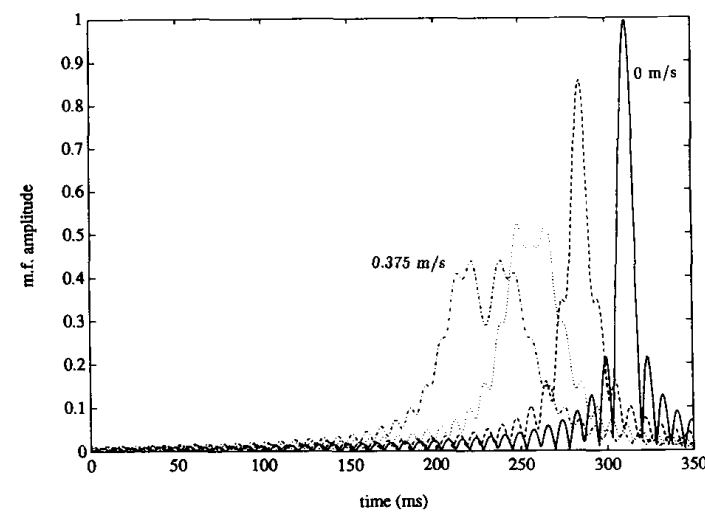

Fig. 8. The Doppler performance of the FM-only linear sweep (boxcar taper) is inferior to that of the TLFM signal. The velocity step (between curves) is the same as Fig. 7. A double peak appears at $v=0.25 \mathrm{~m} / \mathrm{s}$, with very poor performance at greater $v$

section the sidelobes of the boxcar LFM sweeps were shown to be large for $v=0$. Fig. 8 shows that LFM signals also have poor Doppler response with respect to TLFM. The broad multiple nature of the peaks is unacceptable.

The effects of constant Doppler velocities can be eliminated by resampling at the rate $\sigma(1+v / c)$, where $\sigma$ is the sampling rate giving the maximum peak if $v=0$. The relative insensitivity of TLFM peak amplitude to Doppler is most useful if iterative resampling, matched-filtering, and peak searching is unavailable, such as with unsophisticated drifting receivers.

\section{JOINT DOPPLER/ARRIVAL-TTME ESTIMATION}

Since the arrival-time alteration due to Doppler $\tau_{n}^{\prime}$ is a known function of $v, \omega_{c}$ and $b$, and the $b$ dependence is simple, changing the sign of $b$ will simply change the sign of $\tau_{n}^{\prime}$. Careful utilization of this sign-dependence allow simultaneous estimation of $v$ and the true arrival-time of the signal, $t_{t}$.

Consider a signal of duration $T$ with $b=b_{u}>0$ (a frequency-up sweep) followed immediately by a signal of opposite sweep $b=-b_{u}$ having the same mid-frequency $\omega_{c}$ (but not the same $\omega_{o}$ ) and duration $T$. Cross-correlation of the entire signal yields two peaks and is undesirable. Fig. 9 compares the arriving envelopes, $A$, of a Doppler shifted signal with the transmitted envelopes, showing the various important time intervals.

Fig. 10 shows loci of $\tau_{n}^{\prime}$ for each half of the signal, correlated independently, called $\tau_{u}$ and $\tau_{d}$. The data is simulated using a time-dilated version of a Chebyshev-tapered, $120-\mathrm{Hz}$, 30 -s up-sweep followed by a 30 -s down-sweep. The curves are given by

$$
\tau_{u}^{\prime}=\frac{-T \epsilon}{2}-\frac{\omega_{c} \epsilon}{b_{u}}
$$

and

$$
\tau_{d}^{\prime}=\frac{-3 T \epsilon}{2}+\frac{\omega_{c} \epsilon}{b_{u}}
$$

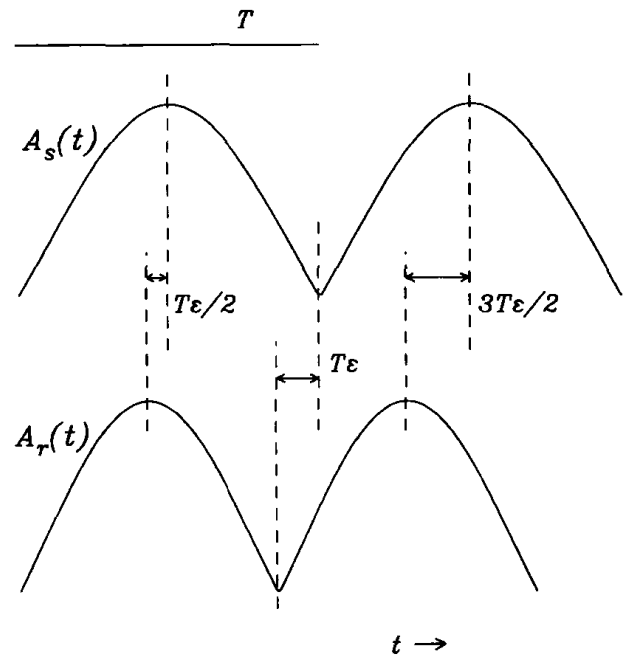

Fig. 9. The transmitted signal envelope $A_{s}$ is compared with the received time-dilated envelope $A_{r}$ for a receiver moving towards to the source, $\epsilon>0$. Each section of the signal (up-sweep followed by down-sweep) has been shortened from $T$ to $T(1-\epsilon)$ duration. Using the arrival time of the initial signal as an index, the average arrival times of the two sweeps differ as shown. The average arrival times of the sweeps appear in the expressions for estimates arrival time versus Doppler velocity, and are called the finite-window corrections.

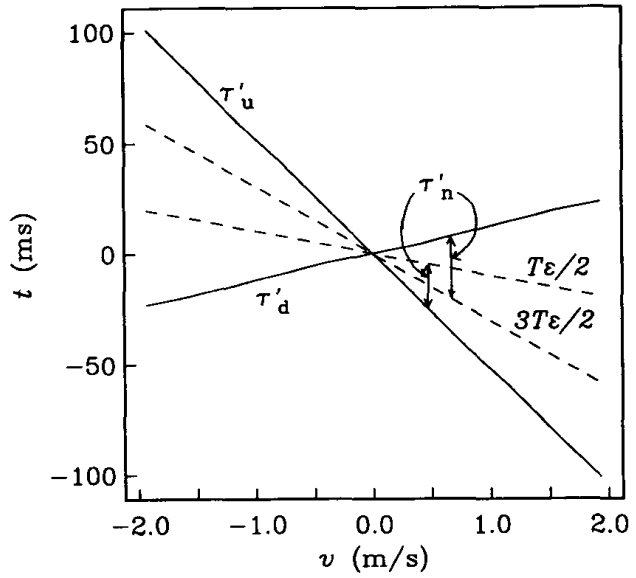

Fig. 10. The loci of the peaks of the matched-filter outputs $\tau_{b}^{\prime}$ for the up sweep $\left(\tau_{u}^{\prime}\right)$ followed by the down sweep $\left(\tau_{d}^{\prime}\right)$. The mean arrival times of each sweep, seen in Fig. 9, are plotted. The difference between the $\tau_{b}^{\prime}$ and the finite-window term (the dashed lines), is equal to the narrow-band Doppler effect $\tau_{n}$, predicted.

The finite-window corrections differ because the down-sweep follows the shortened up-sweep, and are depicted in Fig. 9. The narrow-band contributions differ only in sign.

The velocity can be estimated as

$$
v=\frac{c\left(\tau_{u}^{\prime}-\tau_{d}^{\prime}\right)}{\left(T-2 \omega_{c} / b_{u}\right)}
$$

For multipath arrivals, $\tau_{u}^{\prime}-\tau_{d}^{\prime}$ can be estimated using one or more strong multipaths. Using the estimated $v, \tau_{u}^{\prime}$ (or $\tau_{d}^{\prime}$ ) can 
be determined using (16) or (17), and $t_{t}=\tau_{u}-\tau_{u}^{\prime}=\tau_{d}-\tau_{d}^{\prime}$ can be recovered, where $\tau_{u}$ is the actual arrival-time estimate from the up sweep.

Use of the two consecutive sweeps will give two identical multipath arrival patterns, with one slightly lagging the other. These can be coherently summed with the lag $\tau_{u}^{\prime}-\tau_{d}^{\prime}$ to utilize all of the transmitted energy to give a high-gain ocean impulse response.

\section{SUMMARY}

A family of frequency sweep signals with tapered attenuation at the ends of the band can be used to synthesize pulses with a narrow main peak and low side peaks. The shapes of the main and side peaks are controlled by the tapering. A broad range of taper functions can be used in this TLFM scheme, depending upon signal-noise ratio, expected peak spacing, etc., in order to improve the signal. The functions with optimum properties for this operation are spectral estimation taper functions. Modulation with the square root of a suitable taper function will produce an output pulse shaped like the Fourier transform of the taper.

If the frequency response of the system can be measured, this can be applied to the taper to optimize the output, that is, the applied taper can be adjusted so that the final transmitted spectrum has the desired shape. In principle, one can utilize the entire available frequency range of any equipment, so that maximum attainable time-domain resolution can be achieved.

The TLFM signals provide usable single peaks over a much wider range of Doppler velocities than do untapered linear FM sweeps. This property of TLFM signals makes them an alternative to $\mathrm{M}$-sequences, whose response is attenuated by Doppler shifting unless the sampling frequency is modified. If required, arrival-time variations and apparent timing variations due to Doppler velocities can be separated by combining both up and down sweeps. The peak shift of wide-band TLFM signals due to Doppler velocity is composed of two parts. One part is related to the sweep rate and the center frequency, and results from conventional narrow-band analysis. The second part, a finite-duration correction, is related only to the duration of the signal.

One motivation for this work is the potential high efficiency of quarter-wave resonant tube tomography projectors [6] relative to other types of low frequency $(100-400 \mathrm{~Hz})$ ocean projectors. If a satisfactory method can be developed to tune the tube, such as one tunes a trombone, without introducing vibrations or other losses, pulse synthesis using TLFM sweeps may be ideal.

Tomography experiments to date have used moored sources and receivers. The Doppler properties of TLFM signals may allow cheaper, real-time tomography with small quicklydeployable drifting receivers dangling from surface buoys capable of monitoring their positions. This is conceptually similar to moving-ship tomography [17], but may be less expensive and also allow greater coverage in time and space.

To illustrate the usefulness of TLFM in this context, a test run with a 30 -second $120 \mathrm{~Hz}$ sweep and $-20 \mathrm{~dB}$ signal/noise

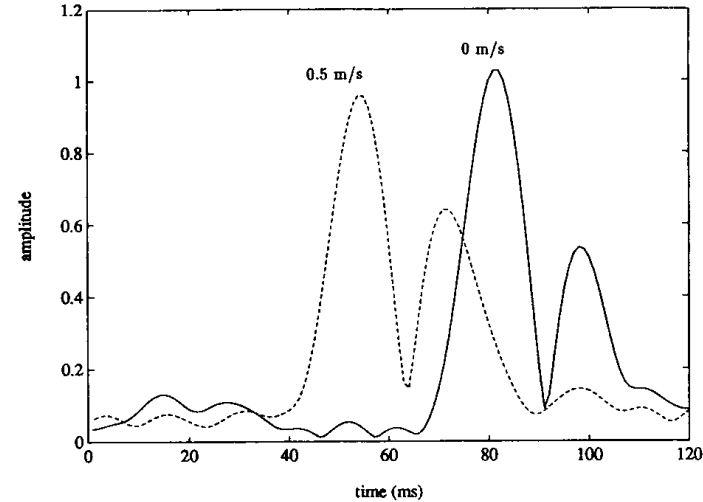

Fig. 11. Synthetic multipath pulse resolution results are very encouraging with both high noise (SNR of $-20 \mathrm{~dB}$ ) and high relative source-receiver velocity. Two simulated arrivals at two Doppler velocities $(0$ and $0.5 \mathrm{~m} / \mathrm{s})$ are shown. A $-20 \mathrm{~dB}$ ripple Chebyshev taper and a 30-s, 120-Hz sweep are employed. The peaks, arriving at 80 and $98 \mathrm{~ms}$, are easily distinguished, with the early arrival shift apparent in the nonzero Doppler case. The slight reduction in peak height at nonzero Doppler is counteracted in this realization by noise effects.

ratio was performed (Fig. 11). Two multipath arrivals at 18 $\mathrm{ms}$ spacing were included, and they are easily identified for the 0 and $0.5 \mathrm{~m} / \mathrm{s}$ Doppler velocities of the simulation, which used the 20-dB ripple Chebyshev taper. The smallness of the Doppler loss at $v=0.5 \mathrm{~m} / \mathrm{s}$ with respect to noise effects is shown by the random variation of peak amplitudes.

\section{ACKNOWLEDGMENT}

Helpful discussions were held with R. Pawlowicz, E. Terray, J. Lynch, J. Preisig, D. Webb, and R. Spindel.

\section{REFERENCES}

[1] P. F. Worcester, B. D. Cornuelle, and R. C. Spindel, "A review of ocean acoustic tomography: 1987-1990," Reviews of Geophysics., Suppl., pp. $557-570,1991$.

[2] J. Spiesberger and K. Metzger, "Basin-scale tomography: A new took for studying weather and climate," J. Geophys. Res., vol. 96, pp. 4869-4889, 1991.

[3] C-S. Chiu and Y. Desaubies, "A planetary wave analysis using the acoustic and conventional arrays in the 1981 ocean acoustic tomography experiment.," J. Phys. Oceanogr., vol. 17, pp. 1279-1287, 1987.

[4] B. Cornuelle, C. Wunsch, D. Behringer, T. Birdsall, M. Brown, R. Heinmiller, R. Knox, K. Metzger, W. Munk, J. Spiesberger, R. Spindel, D. Webb, and P. Worcester, "Tomographic maps of the ocean mesoscale. Part 1: Pure acoustics," J. Phys. Oceanog., vol. 15, pp. 133-152, 1985.

[5] J. H. Miller, J. F. Lynch, and C-S. Chiu, "Estimation of sea surface spectra using acoustic tomography," J. Acoust. Soc. Am., vol. 86, pp. $326-345,1989$.

[6] P. F. Worcester, R. C. Spindel, and B. M. Howe, "Reciprocal acoustic transmissions: Instrumentation for mesoscale monitoring of ocean currents," IEEE J. Oceanic Eng., vol. 10, pp. 123-137, 1985.

[7] S. W. Golomb, Shift Register Sequences. San Francisco: Holden-Day, 1967.

[8] A. W. Rihaczek, Principles of High-Resolution Radar. New York: McGraw-Hill, 1969

[9] R. A. Altes and E. L. Titlebaum, "Bat signals as optimally Doppler tolerant waveforms," J. Acoust. Soc. Am., vol. 48, pp. 1014-1020, 1970.

[10] R. A. Altes, "Some invariance properties of the wide-band ambiguity function," J. Acoust. Soc. Am., vol. 53, pp. 1154-1160, 1973 
[11] Z.-b. Lin, "Wideband ambiguity function of broadband signals," $J$. Acoust. Soc. Am., vol. 83, pp. 2108-2116, 1988.

[12] F. J. Harris, "On the use of windows for harmonic analysis with the discrete Fourier transform," Proc. IEEE, vol. 66, pp. 51-83, 1978.

[13] D. J. Thomson, "Spectrum estimation and harmonic analysis," IEEE Proc., vol. 70, pp. 1055-1096, 1982.

[14] J. N. Little and L. Shure, Signal Processing Toolbox of Use with Matlab, The Math Works, Inc., Natick, MA, 1988.

[15] H. L. Van Trees, Detection, Estimation, and Modulation Theory, Pt. III. New York: Wiley, 1971

[16] T. F. Duda, S. M. Flatte, J. A. Colosi, B. D. Cornuelle, J. A. Hildebrand W. S. Hodgkiss, Jr., P. F. Worcester, B. M. Howe, J. M. Mercer, and R. C. Spindel, "Measured wavefront fluctuations in $1000-\mathrm{km}$ pulse propagation in the Pacific Ocean," J. Acoust. Soc. Am., vol. 92, pp 939-955, 1992.

[17] B. Cornuelle, W. Munk, and P. Worcester, "Ocean acoustic tomography from ships," J. Geophys. Res., vol. 94, pp. 6232-6250, 1989.
Timothy F. Duda received the B.A. degree in physics from Pomona College, Claremont, CA in 1979 and the Ph.D. degree in ocennography from Scripps Institution of Oceanography, University of Califomia, San Diego, in 1986.

He was a research scientist at the University of Califomia, Santa Cruz, and since 1991 has been an Assistant Scientist at Woods Hole Oceanographic Institution. His two primary fields of study are the measurement and characterization of ocean internal waves and microstructure, and ocean acoustic propagation. He has modeled and analyzed fluctuations of ocean acoustic transmissions, has made electromagnetic-type internal-wave measurements, and performedin situ measurement of ocean microstructure.

Dr. Duda is a member of the American Meteorological Society, the American Geophysical Union, and the Acoustical Society of America. 